\title{
La web social en las bibliotecas universitarias de China: análisis de contenidos con mayor impacto
}

\section{The social network of university libraries in China: analysis of the content with greatest impact}

\author{
Manuel Blázquez Ochando \\ http://www.mblazquez.es/
}

Departamento de Biblioteconomía y Documentación de la Facultad de Ciencias de la Documentación de la Universidad Complutense de Madrid

\author{
Yue Wang \\ http://www.mblazquez.es/ \\ Departamento de Documentación, Universidad Carlos III de Madrid
}

\begin{abstract}
Resumen
El rápido desarrollo de las redes sociales en todo el mundo ha propiciado una explosión demográfica del número de perfiles de bibliotecas universitarias que pretenden acercar sus servicios y actividades a un público más alejado, por las facilidades que ofrece la Web para encontrar información y recursos. A pesar de todo, las bibliotecas universitarias desarrollan estrategias para acercar a los usuarios y fomentar el uso de los fondos documentales en los espacios físicos y digitales. En este caso, se estudian los contenidos de mayor impacto de los 50 perfiles más importantes de bibliotecas universitarias de China, en la red social Weibo, destacada por su volumen de tráfico y usuarios. El estudio relaciona los datos de fecha de creación, seguidores, publicaciones e impacto para determinar qué actividades y contenidos publicados tienen mayor relevancia entre los usuarios, de forma que puedan ser utilizados en las bibliotecas universitarias españolas para perfeccionar su interacción social. Por otra parte, también se proporcionan datos sobre el posicionamiento de las bibliotecas universitarias analizadas en el ranking de universidades de China, su tasa de interacción, el tipo de contenidos más abundante y que mayor número de comentarios y re-publicaciones obtiene.
\end{abstract}

\section{Palabras clave}

Redes sociales, Bibliotecas universitarias, China, Weibo, Análisis de contenidos, Análisis de impacto 


\begin{abstract}
The rapid development of social networks around the world has led to a population explosion in the number of profiles of university libraries that attempt to bring his services and activities to the farther public by the facilities offered by the Web to find information and resources. Nevertheless, the university libraries develop strategies to approach users and encourage the use of the documentary collections in the physical and digital spaces. In this case, has been studied the contents of the 50 profiles of major university libraries in China that has the greatest impact in the social network Weibo, which is highlighted by the volume of traffic and users. The study relates the data from creation date, followers, publications and impact to determine what activities and contents published are relevant most among the users, so they can be used in the Spanish university libraries to improve their social interaction. On the other hand, there are also provided the data about the positioning of the university libraries in the ranking of universities in China, the rate of interaction, the most abundant type of content and the largest number of comments and re-publications that has been obtained.
\end{abstract}

\title{
Keywords
}

Social networks, University libraries, China, Weibo, Content analysis, Impact analysis

Recibido: 04/07/2016

Aceptado: 16/10/2016

DOI: http:/ / dx.doi.org/10.5557/IIMEI7-N13-145165

Descripción propuesta: BLÁZQUEZ OCHANDO, Manuel; WANG, Yue, 2016. La web social en las bibliotecas universitarias de China: análisis de contenidos con mayor impacto. Métodos de Información [en línea], 7(13), pp. 145-165.

https://creativecommons.org/licenses/by/4.0/

\section{Introducción}

La relación entre bibliotecas y redes sociales tienen su origen en el concepto biblioteca 2.0. Según Margaix (2007) las redes sociales pueden ayudar a los catálogos en línea a convertirse en herramientas para la difusión social de las colecciones bibliográficas. Ello plantea una oportunidad de identificar mejor a los usuarios, considerar sus necesidades de información y homogeneizarlas. 
Este enfoque demuestra la importancia de las redes sociales para unir biblioteca, información, recursos, intereses, usuarios, actividades y servicios en un mismo punto de encuentro digital.

Por su parte Milstein (2009) se centra en los potenciales usos de la red Twitter para las bibliotecas. Twitter ofrece servicios de publicación de mensajes cortos que pueden ser aprovechados por los bibliotecarios para difundir informaciones puntuales, precisas e instantáneas a los usuarios reales y potenciales de la biblioteca, ofreciendo una mayor difusión. En relación a bibliotecas universitarias, destaca el enfoque de Hansen, Nowlan y Winter (2012) que explica la experiencia de uso de la red social Pinterest en la biblioteca de la Universidad de Regina. Se abordó la creación de un centro de interacción para la comunidad universitaria a través de las imágenes clasificadas temáticamente por sus usuarios. Ello permitía mostrar, a golpe de imagen, las portadas e imágenes de los documentos más notables, recibidos por la biblioteca y ofrecer una difusión novedosa y atractiva para el usuario que fomentó su participación, uso y demanda de los recursos. También coincide De Volder y Carrizo (2014) en los usos y adaptaciones de Pinterest en las bibliotecas de España e Iberoamérica, llegando a la conclusión de que sus funciones más importantes son la difusión de colecciones, la presentación de servicios en línea y la propuesta de actividades vinculadas con la formación de usuarios y la recuperación de recursos web-bibliográficos.

En China, sin embargo, las redes sociales utilizadas por las bibliotecas son diferentes, debido a la idiosincrasia cultural que determina el funcionamiento de sus instituciones universitarias y a la concepción de los servicios de comunicación social en Internet. Por ejemplo Qian y Wang (2008) expone el caso de la red social Tencent $Q Q$ en la Biblioteca de la Universidad Normal de Xinzhou, indicando que cada departamento de la biblioteca (técnica de la información, préstamo, adquisición, publicaciones periódicas, gestión bibliotecaria), presentan información relevante para el usuario. En concreto, directorios de enlaces y recursos de acceso a perfiles y sitios web de la biblioteca, facultades y universidad, información sobre las características de los servicios de préstamo y sus correspondientes avisos, desideratas y difusión de artículos más relevantes en las revistas científicas de cada área de conocimiento. 
También destaca la red Douban especializada en libros, música y películas (Si, 2008), que proporciona una plataforma de trabajo y facilidades de adaptación mayores, debido a que la comunicación en interacción se basa en la industria cultural. Por este motivo Zhao (2009) analiza la relación entre la red social Douban y las bibliotecas, llega a la conclusión de que su éxito se debe al registro de las filiaciones y gustos del usuario, así como el registro de las elecciones intelectuales, que quedan plasmadas en sus listas de deseos y favoritos. Ello supone una oportunidad comercial para las industria cultural china, pero también para la biblioteca, que puede adaptarse mejor a las necesidades intelectuales, culturales y científicas de un público más diverso. También destaca los problemas legales y diatribas que pueden plantear redes como Douban en relación a la protección de los derechos de autor, propiedad intelectual, derechos de explotación, que entran en conflicto con la necesidad de abrir el conocimiento a la sociedad.

Es reseñable el estudio de Li y Gao (2010) que propone el empleo de la red Tencent QQ para crear foros de consultas y respuestas en el marco de las bibliotecas, aglutinando distintos tipos de usuarios, profesores, investigadores y estudiantes, con los que existe una interacción o reciprocidad en la comunicación. Según Yang, Zhang y Zou (2010) ello da lugar al concepto LibSNS o lo que es lo mismo biblioteca social. Su objeto se enmarca en la relación del usuario con el medio documental, con la intención de anticiparse a las necesidades de información.

Otro enfoque es el análisis comparativo entre redes sociales de Asia y Occidente en el que Peng (2012) destaca las diferencias de interacción de Facebook y su contrapartida china RenRen como plataformas para la difusión de investigaciones científicas en el marco de las bibliotecas universitarias. Según los estudios de Shi y Qu (2014) sitúan ésta relación entre biblioteca, universidad y red social en un entorno más personalizado denominado redes sociales multiservicio. Una red social multiservicio consiste en una plataforma de comunicación que proporciona los servicios de microblogging, muro, chat, blog y red social integrados. Un ejemplo de ello es el perfil de la Biblioteca de la Universidad de Nanjing, que utiliza la red WeChat, desde el año 2013, para facilitar el servicio de préstamo, consulta de OPAC, difusión bibliográfica y gestión de adquisiciones bajo demanda. Para lograrlo, la red social ha desarrollado un módulo específicamente diseñado para dinamizar estos 
procesos en la biblioteca y ha demostrado aumentar la interacción entre los usuarios y la biblioteca de forma notable.

Pero la red social más importante en cuanto a número de usuarios activos, tráfico e influencia en las bibliotecas universitarias de China es Weibo (Wang 2010). Por este motivo, los esfuerzos de investigación se vienen centrando en la evolución de sus perfiles sociales y en el nivel de penetración entre los usuarios de las bibliotecas (Huang 2012). De hecho, según las consultas realizadas en Weibo, a finales del año 2015 se detectaron 635 perfiles de bibliotecas, de las cuales 216 eran de tipo universitario. Dicho de otra forma, la mayoría de las universidades de China está representada en Weibo (Tang et al. 2013) y ello justifica que sea el entorno de análisis elegido para el presente estudio.

Este trabajo tiene el objetivo de determinar cuáles son los perfiles más relevantes de las bibliotecas universitarias de China en la red social Weibo. Una vez seleccionados obtener datos sobre el número de seguidores, tasa de interacción, contenidos con mayor impacto, clasificación y análisis cualitativo. El estudio de los contenidos puede ayudar a otras bibliotecas en la aplicación de nuevas ideas que dinamicen la relación con sus usuarios, recreando las experiencias, actividades y recursos.

\section{Metodología}

La investigación de la web social de las bibliotecas universitarias de China se ha orientado al análisis de los contenidos que más impacto han generado entre los usuarios. Para lograr este objetivo ha sido necesario escoger entre diversas redes sociales como Renren, Tencent QQ, Q-Zone, Wechat, Douban o Zhihu, la más adecuada. En este caso se ha elegido la red social Weibo por ser la más utilizada por usuarios y bibliotecas universitarias de China, permitiendo además la verificación de sus perfiles. Usando las herramientas de consulta avanzada en Weibo es posible conocer los 50 perfiles de bibliotecas universitarias con mayor número de seguidores (Tabla 1). 


\begin{tabular}{|c|c|c|c|}
\hline ID & Biblioteca & ID & Biblioteca \\
\hline 1 & $\begin{array}{l}\text { Biblioteca de la Universidad } \\
\text { Tsinghua }\end{array}$ & 26 & Biblioteca de UNS \\
\hline 2 & $\begin{array}{l}\text { Biblioteca de la Universidad de } \\
\text { Wuhan }\end{array}$ & 27 & Biblioteca de UNH \\
\hline 3 & Biblioteca de la UX & 28 & $\begin{array}{l}\text { Biblioteca de la Universidad de } \\
\text { Tecnología de Anhui }\end{array}$ \\
\hline 4 & $\begin{array}{l}\text { Biblioteca de la Universidad de } \\
\text { Pekín }\end{array}$ & 29 & Biblioteca de UNL \\
\hline 5 & $\begin{array}{l}\text { Biblioteca de la Universidad } \\
\text { Fudan }\end{array}$ & 30 & $\begin{array}{l}\text { Weibo Oficial de la Biblioteca de la } \\
\text { Universidad de Jinan }\end{array}$ \\
\hline 6 & Biblioteca de la UEEG & 31 & Biblioteca de UNS \\
\hline 7 & $\begin{array}{l}\text { Biblioteca de la Universidad } \\
\text { Tongji }\end{array}$ & 32 & $\begin{array}{l}\text { Biblioteca de la Universidad de } \\
\text { Shanghái Jiao Tong }\end{array}$ \\
\hline 8 & $\begin{array}{l}\text { Biblioteca de la Universidad de } \\
\text { Chongqing }\end{array}$ & 33 & $\begin{array}{l}\text { Biblioteca de la Universidad Médico } \\
\text { del Sur }\end{array}$ \\
\hline 9 & $\begin{array}{l}\text { Biblioteca de la Universidad de } \\
\text { Sichuan }\end{array}$ & 34 & $\begin{array}{l}\text { Biblioteca de la Universidad Popular } \\
\text { de Seguridad Pública de China }\end{array}$ \\
\hline 10 & Biblioteca de la UJN & 35 & $\begin{array}{l}\text { Biblioteca del Colegio Tan Kah Kee } \\
\text { de la Universidad de Xiamen }\end{array}$ \\
\hline 11 & $\begin{array}{l}\text { Biblioteca de la Universidad } \\
\text { Normal del Este de China }\end{array}$ & 36 & $\begin{array}{l}\text { Biblioteca de la Universidad de } \\
\text { Ciencia y Tecnología de Kunming }\end{array}$ \\
\hline 12 & $\begin{array}{l}\text { Biblioteca Médica de la } \\
\text { Universidad de Pekín }\end{array}$ & 37 & $\begin{array}{l}\text { Biblioteca de la Universidad de } \\
\text { Zhejiang Gongshang }\end{array}$ \\
\hline 13 & $\begin{array}{l}\text { Com. de Lago Sur de la Bib. Inst. } \\
\text { de Ciencia y Tecnología de Hunan }\end{array}$ & 38 & $\begin{array}{l}\text { Biblioteca de la Universidad de } \\
\text { Tecnología de Beijing }\end{array}$ \\
\hline 14 & $\begin{array}{l}\text { Biblioteca de la Universidad de } \\
\text { Economía de Hubei }\end{array}$ & 39 & $\begin{array}{l}\text { Biblioteca de la Universidad de Xi'an } \\
\text { Jiaotong }\end{array}$ \\
\hline 15 & $\begin{array}{l}\text { Biblioteca de la Universidad de } \\
\text { Nanjing }\end{array}$ & 40 & $\begin{array}{l}\text { WO de la Biblioteca de la } \\
\text { Universidad de Qingdao Binhai }\end{array}$ \\
\hline 16 & $\begin{array}{l}\text { Biblioteca de la Universidad de } \\
\text { Minería y Tecnología de China }\end{array}$ & 41 & $\begin{array}{l}\text { Biblioteca de la Universidad de } \\
\text { Zhengzhou }\end{array}$ \\
\hline 17 & $\begin{array}{l}\text { Biblioteca de la Universidad de } \\
\text { Shenzhen }\end{array}$ & 42 & $\begin{array}{l}\text { Biblioteca de la Universidad de } \\
\text { Hainan }\end{array}$ \\
\hline 18 & Biblioteca de UNN & 43 & $\begin{array}{l}\text { Biblioteca de la Universidad de } \\
\text { Tecnología y Negocios de Beijing }\end{array}$ \\
\hline 19 & Biblioteca de UNM & 44 & $\begin{array}{l}\text { Biblioteca de la Universidad } \\
\text { Aeronáutica y Astronáutica de } \\
\text { Beijing }\end{array}$ \\
\hline 20 & $\begin{array}{l}\text { Biblioteca de la Universidad de } \\
\text { Finanzas y Economía del } \\
\text { Suroeste }\end{array}$ & 45 & $\begin{array}{l}\text { Biblioteca de la Universidad de } \\
\text { Petróleo de China }\end{array}$ \\
\hline
\end{tabular}




\begin{tabular}{|c|l|c|l|}
\hline 21 & $\begin{array}{l}\text { Biblioteca de Humanidad de } \\
\text { Tsinghua }\end{array}$ & 46 & $\begin{array}{l}\text { Biblioteca de la Universidad de } \\
\text { Finanzas de Guangdong }\end{array}$ \\
\hline 22 & $\begin{array}{l}\text { Biblioteca de la Universidad del } \\
\text { Sureste }\end{array}$ & 47 & $\begin{array}{l}\text { Biblioteca de la Universidad Normal } \\
\text { de Hebei }\end{array}$ \\
\hline 23 & $\begin{array}{l}\text { Biblioteca de la Universidad de } \\
\text { Finanzas y Economía de } \\
\text { Shanghai }\end{array}$ & 48 & $\begin{array}{l}\text { Biblioteca de la Universidad de } \\
\text { Tecnología de Hefei }\end{array}$ \\
\hline 24 & Biblioteca Zhongkai & 49 & $\begin{array}{l}\text { Biblioteca de la Universidad de CC. } \\
\text { Política y Derecho del Suroeste }\end{array}$ \\
\hline 25 & $\begin{array}{l}\text { Biblioteca de la Universidad } \\
\text { Renmin de China }\end{array}$ & 50 & $\begin{array}{l}\text { Biblioteca de la Universidad de } \\
\text { Economía y Derecho de Zhongnan }\end{array}$ \\
\hline
\end{tabular}

Tabla 1. Referencia de perfiles de bibliotecas universitarias analizadas

Para cada biblioteca universitaria se obtuvieron datos relativos al número de contenidos publicados, comentarios, re-publicaciones, fecha de creación, posición en el ranking de universidades y tasa de interacción. Esto fue posible mediante el uso de la herramienta métrica Tfengynn.com desarrollada conjuntamente por Weibo y Baidu, el principal buscador de China. Ésta información puede ser consultada en el anexo 1 del trabajo.

El siguiente paso consiste en abordar el estudio temático y cualitativo de los contenidos publicados. Se recuperaron las 5 publicaciones más relevantes para los usuarios en cada perfil de la lista, obteniendo un total de 250 publicaciones que serían clasificadas, de acuerdo a palabras clave, agrupadas en torno a categorías, que ayudasen a determinar los grupos de informaciones, noticias, actividades y contenidos de mayor interés.

\section{Resultados}

El análisis de los datos obtenidos ${ }^{1}$ demuestra que el $64 \%$ de los perfiles de las bibliotecas universitarias en la red social Weibo fueron creados en el año 2011. Es una cifra elevada si se compara con el resto de años en los que se fundaron el resto de perfiles desde el año 2009 hasta el 2014 (Figura 1). Por otra parte la Biblioteca de la Universidad de Chongqing y de la UX son las primeras bibliotecas en crear el perfil social en el año 2009. También se advierte que después del año 2011 se produce una tendencia descendente en el número de perfiles, lo que significa que la mayor parte ya habían sido creados. 


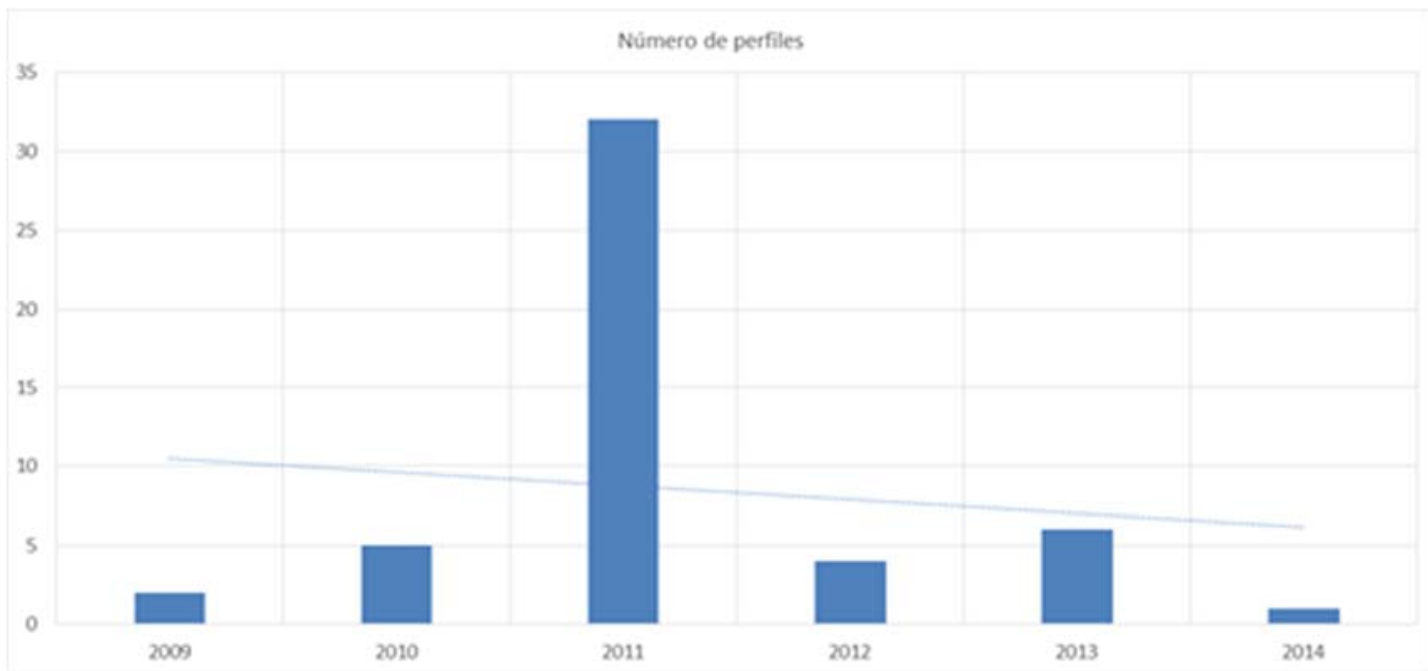

Figura 1. Año de fundación de los perfiles de las bibliotecas universitarias en Weibo

En relación al número de seguidores y publicaciones, se confirma que no existe una correlación clara (Figura 2). De hecho las bibliotecas que más publicaciones tienen no siempre tienen un número semejante de seguidores. Esto puede explicar que la pertinencia, calidad y relevancia de los contenidos son factores más importantes para hacer crecer las comunidades sociales.

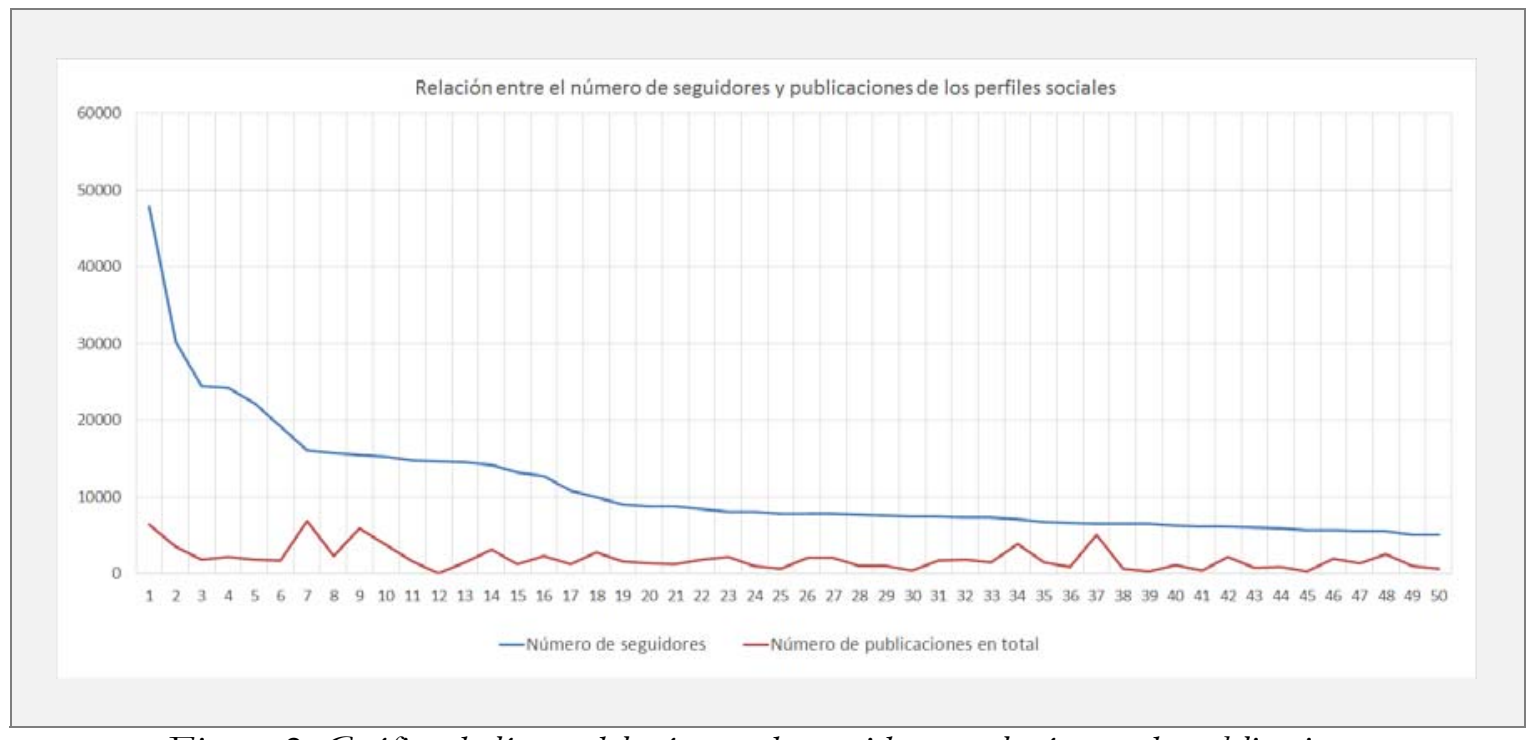

Figura 2. Gráfico de líneas del número de seguidores y el número de publicaciones Atendiendo a la muestra y a fecha de 23 de agosto del 2015, el número de seguidores de los perfiles varía desde 5.000 hasta 47.000 y el número de publicaciones desde 86 hasta 6.818 como máximo. Un caso llamativo es la Biblioteca Médica de la Universidad de Pekín que registrando únicamente 86 publicaciones, se sitúa en el puesto número 12 por cantidad de seguidores. 
Algunas razones que explican estos datos se encuentran en que el perfil social de la Universidad de Pekín fue creado en diciembre del año 2013. Esto significa que en el periodo analizado no pudo desarrollar tantas publicaciones como aquellas bibliotecas más veteranas en la implantación de su sede social. Otra razón que explica el rápido crecimiento de seguidores es la relación de la Biblioteca de medicina de la Universidad de Pekín y el perfil social del Hospital de la Universidad de Pekín, que dispone de 1.084.080 seguidores. Si se compara con el número de seguidores de la Universidad de Pekín (342.992) y el correspondiente al de la Biblioteca de la Universidad de Pekín (25.553) se puede afirmar que son cifras ridículas. Por tanto el crecimiento se debe al masivo redireccionamiento, casi natural, del tráfico de lectura de contenidos, que llevan a cabo los usuarios entre perfiles Weibo, que están directamente relacionados. Esta afirmación puede confirmarse al detectar que existen artículos o publicaciones en el perfil de la Biblioteca de la Universidad de Pekín que aluden directamente al perfil de la Biblioteca Médica de la Universidad.

Las 50 bibliotecas de la muestra coinciden con las universidades mejor posicionadas de China según el Chinese University Ranking CUAA. El 40\% de los perfiles se encuentran entre las primeras 50 universidades de China y un porcentaje similar corresponde a las siguientes 150 universidades. Esto significa que las mejores bibliotecas universitarias ofrecen servicios de información y referencia, atención al usuario y difusión de contenidos a través de las redes sociales.

Atendiendo al régimen de publicaciones, se estima que el 60\% de los perfiles publican 1 contenido diario, siendo la Biblioteca de la Universidad de Tongji la que más contenidos publica, con un ratio de 4,4 por día. También se advierte que 30 perfiles tienen al menos 1 comentario en cada publicación y en todos los casos fueron compartidas o re-publicadas como mínimo en una ocasión. En este sentido la biblioteca que mejor tasa de interaccion ${ }^{2}$ obtiene es la Biblioteca de la Universidad Popular de Seguridad Pública de China, con un $0,26 \%$.

Después de analizar las 250 publicaciones con mayor impacto de la muestra, se ha estudiado su temática y se observa que los avisos de la biblioteca y los horarios son los asuntos más publicados y que mayor impacto generan. Ello 
representa un 20\% de publicaciones, frente al 13\% destinado a las actividades culturales y académicas. Éstas son por ejemplo la participación en coloquios y foros sobre los libros, conferencias y temas de interés para la formación académica para la obtención de premios y reconocimientos, actividades relativas a la re-publicación y difusión de las publicaciones del perfil de la biblioteca para obtener gratificaciones en forma de libros, o la participación de actividades de voluntariado de la biblioteca para conocer mejor sus servicios y obtener ventajas de préstamo, reserva de documentos y puestos de lectura o de trabajo.

\begin{tabular}{|c|c|c|c|}
\hline ID & Grupo temático & $\begin{array}{l}\text { Frecuencia } \\
\text { de } \\
\text { aparición }\end{array}$ & Porcentaje \\
\hline 1 & $\begin{array}{l}\text { Avisos de la biblioteca, horarios, apertura y } \\
\text { cierres temporales del centro, normativa de la } \\
\text { biblioteca, cuestiones de carácter general, } \\
\text { informes }\end{array}$ & 57 & $20 \%$ \\
\hline 2 & $\begin{array}{l}\text { Actividades culturales y académicas de la } \\
\text { biblioteca, conferencias, exposiciones, concursos } \\
\text { y premios }\end{array}$ & 37 & $13 \%$ \\
\hline 3 & $\begin{array}{l}\text { Atención al usuario, preguntas y respuestas, } \\
\text { sugerencias, carnet de la biblioteca, sala de lectura }\end{array}$ & 35 & $12 \%$ \\
\hline 4 & $\begin{array}{l}\text { Desideratas, adquisición, intercambio y venta de } \\
\text { libros y documentos, préstamo y devolución de } \\
\text { libros, servicios bibliotecarios, información y } \\
\text { referencia, biblioteca digital, OPAC }\end{array}$ & 26 & $9 \%$ \\
\hline 5 & $\begin{array}{l}\text { Fuentes de información, recursos de información } \\
\text { científica y académica, bases de datos, CNKI, } \\
\text { revistas científicas, artículos científicos }\end{array}$ & 25 & $9 \%$ \\
\hline 6 & $\begin{array}{l}\text { Noticias de la biblioteca o de la universidad a la } \\
\text { que pertenece o sobre otras bibliotecas y temas } \\
\text { afines, actos de graduación }\end{array}$ & 24 & $8 \%$ \\
\hline 7 & $\begin{array}{l}\text { Contenidos relacionados con fotografía y } \\
\text { publicación de imágenes, materiales audiovisuales } \\
\text { y películas }\end{array}$ & 20 & $7 \%$ \\
\hline 8 & $\begin{array}{l}\text { Formación de usuarios, cursos de aprendizaje y } \\
\text { enseñanza de instrumentos, herramientas y }\end{array}$ & 16 & $5 \%$ \\
\hline
\end{tabular}




\begin{tabular}{|c|c|c|c|}
\hline & $\begin{array}{l}\text { servicios de información y documentación de la } \\
\text { biblioteca, cursos de búsqueda en catálogos, } \\
\text { bibliografías }\end{array}$ & & \\
\hline 9 & $\begin{array}{l}\text { Literatura, teatro, poesía, refranes, historia, } \\
\text { humanidades }\end{array}$ & 15 & $5 \%$ \\
\hline 10 & $\begin{array}{l}\text { Noticias no relacionadas con el entorno } \\
\text { bibliotecario o universitario }\end{array}$ & 11 & $4 \%$ \\
\hline 11 & $\begin{array}{l}\text { Metodología de la investigación, tesis doctorales, } \\
\text { publicación de trabajos científicos }\end{array}$ & 10 & $3 \%$ \\
\hline 12 & Fomento de la lectura, lecturas recomendadas & 9 & $3 \%$ \\
\hline 13 & $\begin{array}{l}\text { Empleo en la biblioteca, voluntariado de alumnos } \\
\text { para ayudantías en la biblioteca }\end{array}$ & 7 & $2 \%$ \\
\hline
\end{tabular}

Tabla 2. Contenidos publicados según grupos temáticos

El tercer grupo temático, con un 12\% de publicaciones, corresponde a los asuntos de atención al usuario, sus preguntas y sugerencias, carnet de identificación en la biblioteca y problemas de gestión de los puestos en la sala de lectura. Con porcentajes inferiores al 10\% se encuentran los grupos de servicios de la biblioteca, fuentes de información, noticias de la biblioteca, formación de usuarios, fomento de la lectura, metodología de la investigación y empleo en la biblioteca (Tabla 2).

\section{Conclusiones}

La fecha clave de popularización de Weibo como red social para las bibliotecas universitarias de China fue el año 2011. De las 50 bibliotecas universitarias más destacadas, la mayoría ya disponía de un perfil social con 4 años de funcionamiento, proporcionándoles experiencia en el uso de este tipo de sistemas de comunicación. Ello ha permitido a estas bibliotecas universitarias la posibilidad de cambiar y ajustarse a las necesidades de la sociedad actual en general y a la de los estudiantes universitarios en particular.

El número de seguidores de un perfil social en Weibo depende de la calidad de las publicaciones y no de la cantidad. No existe una correlación clara de este hecho. Por tanto resultan determinantes otros factores como la utilidad de la información para el usuario, la forma de presentar las actividades, la 
creación de incentivos y ventajas por la participación en las mismas. También influyen notablemente las tácticas de redirección de tráfico de usuarios entre perfiles de institucionales distinguiéndose alianzas y vínculos de filiación profesionales.

Se puede afirmar que las bibliotecas de las mejores universidades de China tienen un perfil más activo en la red social Weibo y ello sirve como método para captar nuevos usuarios y lectores. Esto significa que las redes sociales son el nuevo acceso a la biblioteca y medio de difusión de sus servicios. Otro factor que sirve para atraer nuevos seguidores al perfil de la biblioteca es la reputación de la universidad, logrando en tal caso acercar los alumnos más talentosos y participativos, como se demuestra tras analizar los datos del Ranking de Universidades de China CUAA.

La tasa de interacción de los usuarios en relación a los contenidos publicados por las bibliotecas universitarias permite afirmar, que la media de publicaciones diarias recomendadas no debería sobrepasar los 2 o 3 contenidos. Esto se explica al comprobar que los perfiles con mayor número de seguidores son aquellos que reproducen éste patrón. También se confirma que la publicación programada en los periodos de máxima audiencia, ayuda a profundizar en el impacto de los seguidores y aumentar la difusión de la información. Sin embargo aumentar el régimen de publicaciones puede provocar el efecto contrario propiciando una saturación innecesaria. De hecho la biblioteca con más publicaciones no es la que más seguidores tiene y tampoco es la que más comentarios e impacto genera.

Los contenidos con mayor interacción en los perfiles sociales, corresponden a los de tipo horario, avisos, normativa de la biblioteca, hechos y cifras, siendo los más visitados y comentados. Las actividades académicas, conferencias exposiciones, actividades culturales, concursos, coloquios, foros y atención al usuario, representan el $45 \%$ de todos los contenidos publicados. Los servicios de la biblioteca, fuentes y recursos de información, bases de datos y noticias de la biblioteca constituyen un 33\% del total. Los asuntos relativos a formación de usuarios están presentes en el $5 \%$ de las publicaciones y metodología de la investigación, así como fomento de la lectura, con un 3\% cada uno. Estos datos demuestran que la red social potencia el contacto con el 
usuario, su atención y participación en actividades, por encima de otros contenidos.

\section{Notas}

1 Los datos obtenidos están disponibles para su consulta, revisión y análisis en las siguientes direcciones:

Anexo 1. Bibliotecas universitarias analizadas. https://goo.gl/QvfvCd

Anexo 2. Selección de las 250 publicaciones con mayor impacto. https://goo.gl/pu1qZN

Anexo 3. Desglose de temáticas y su frecuencia de aparición. https://goo.gl/EJhYdu

2 La tasa de interacción es calculada a partir del número total de comentarios y republicaciones diarias divido por el número total de seguidores del perfil.

\section{Bibliografía}

CHINESE UNIVERSITIES ALUMNI ASSOCIATION, 2015. Ranking de los primeros 700 universidades de China. En: Chinese Universities Alumni Association [en línea]. [Consulta: 23 agosto 2015]. Disponible en: http://www.cuaa.net/cur/2015/index_700/

DE VOLDER, C. y CARRIZO, V. I., 2014. Construcción de colecciones sociales en bibliotecas iberoamericanas: el caso Pinterest. Palabra clave, 4(1), 33-47. ISSN 1853-9912

HANSEN, K., NOWLAN, G. y WINTER, C., 2012. Pinterest as a Tool: Applications in Academic Libraries and Higher Education. Canadian Journal of Library and Information Practice and Research, 7(2). ISSN 1911-9593

HUANG, S., 2012. Analysis of library use microblog features and Development Strategy = 图书馆微博使用特征及发展策略研究. Journal of Academic Libraries, (1), 78-83. ISSN 1002-1027 
LI, L. y GAO, F., 2010. Application of Tencent QQ Group in Virtual Reference Service in University Libraries = QQ 群在高校图书馆虚拟参考咨询服务中的应 用. Journal of Library and Information Science in Agriculture, 22(1), 65-69. DOI:10.13998

MARGAIX, D., 2007. El OPAC Social, el catálogo en la Biblioteca 2.0. Aplicación y posibilidades en las Bibliotecas Españolas. E-información, integración y rentabilidad en un entorno digital: $10^{a}$ Jornadas Españolas de Documentación (FESABID 07), 199-205

MILSTEIN, S., 2009. Twitter for Libraries (and librarians). Computers in libraries, 29(5), 17-18. ISSN 1041-7915

PENG, Y., 2012. A Comparative Study on the Application of SNS by University Libraries at Home and Abroad = 国内外高校图书馆社交网络应用比较研究. Research on Library Science, 17, 79-81

QIAN, W. y WANG, W., 2008. Talking about the application of Tencent QQ Software in Universy Library = 浅谈腾讯QQ软件在高校图书馆中的应用. SciTech Information Development \& Economy, 18(33), 28-30

SHI, M. y QU, X., 2014. WeChat Marketing Strategy in Academis Libraries = 高校 图书馆官方微信经营策略研究———南京大学图书馆为例. Journal of Academic Libraries, (5), 79-85

SI, J., 2008. When library Encounters Douban = 当图书馆遇到豆瓣. Library Journal, 27(3), 15-18. DOI 10.1366

TANG, Q., YUAN, Y. y LIU, Z., 2013. A survey on the Current Situation of College Libraries Microblog Service in China - Taking Authenticated Users on Sina Weibo for Example = 我国高校图书馆微博服务现状调查研究---以新浪认证 用户为例. Journal of Academic Libraries, (3), 97-103

TFENGYUN, 2015. Tfengyun [en línea]. Pekín: BOOSEN. [Consulta: 16 agosto 2015]. Disponible en: http://www.tfengyun.com/ 
WANG, M., 2010. La situación actual de Weibo en las bibliotecas de = 国内图书馆 微博应用现状及建议. Research on Library Science, (12), 37-41

YANG, J., ZHANG, C. y ZOU, Y., 2010. On the Construction of Lib-SNS = 关于 图书馆社交网络(LibSNS)构建的研究. Research on Library Science, (15), 27-31

ZHAO, L., 2009. The seccess of Douban and the Development of Library $=$ 豆瓣 的成功与图书馆的发展. Journal of Modern Information, 29(9), 42-44 


\section{Anexo}

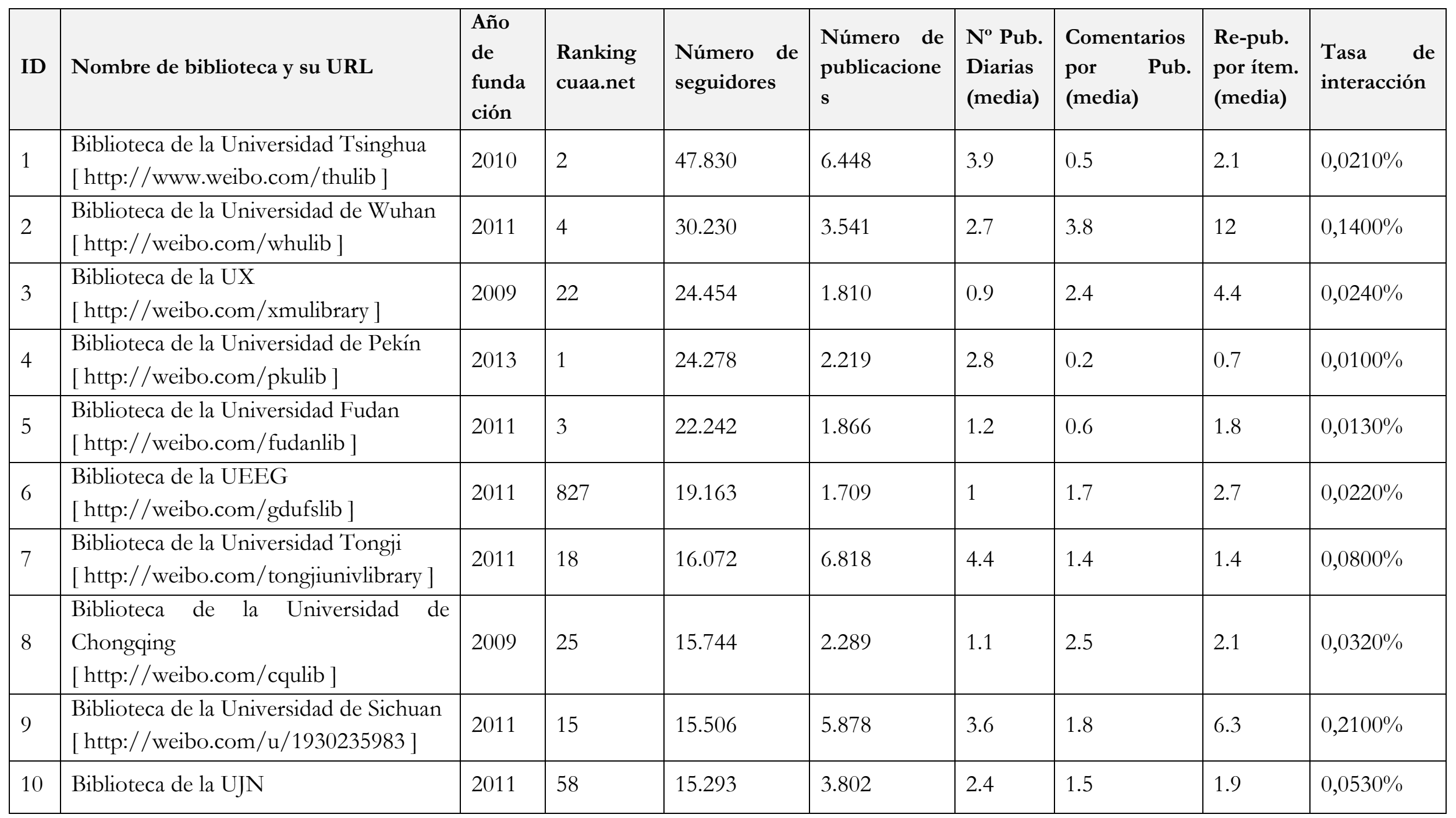




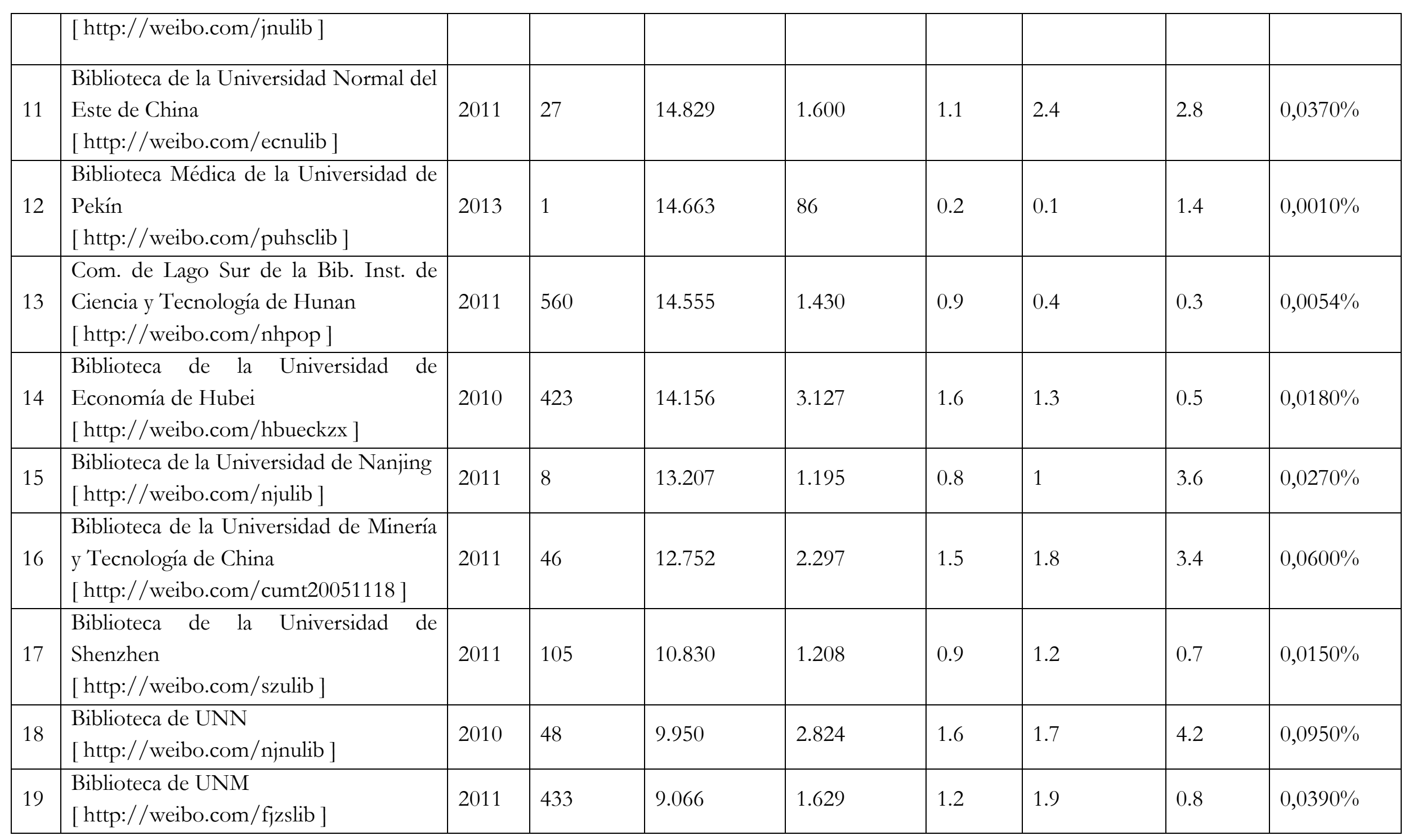




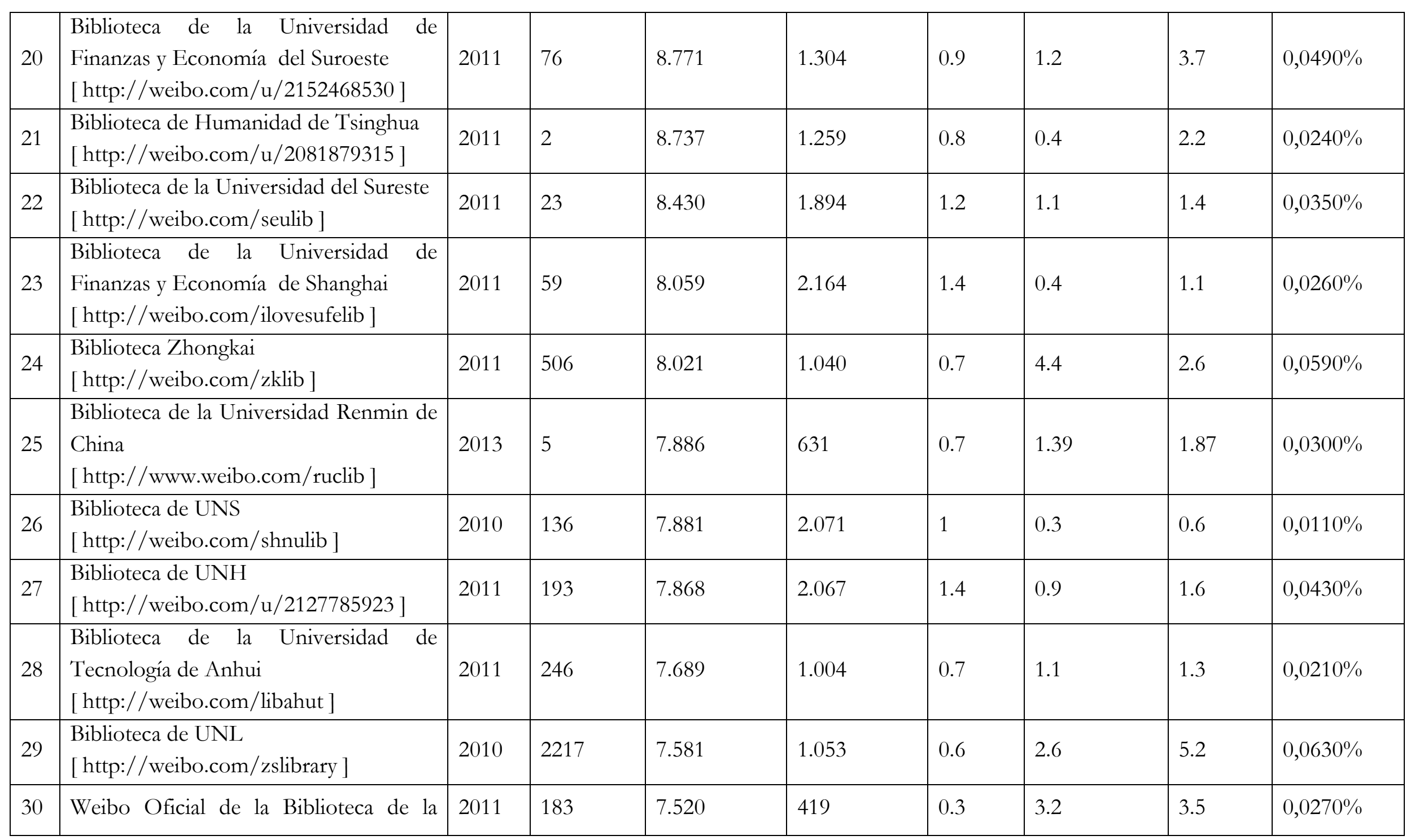




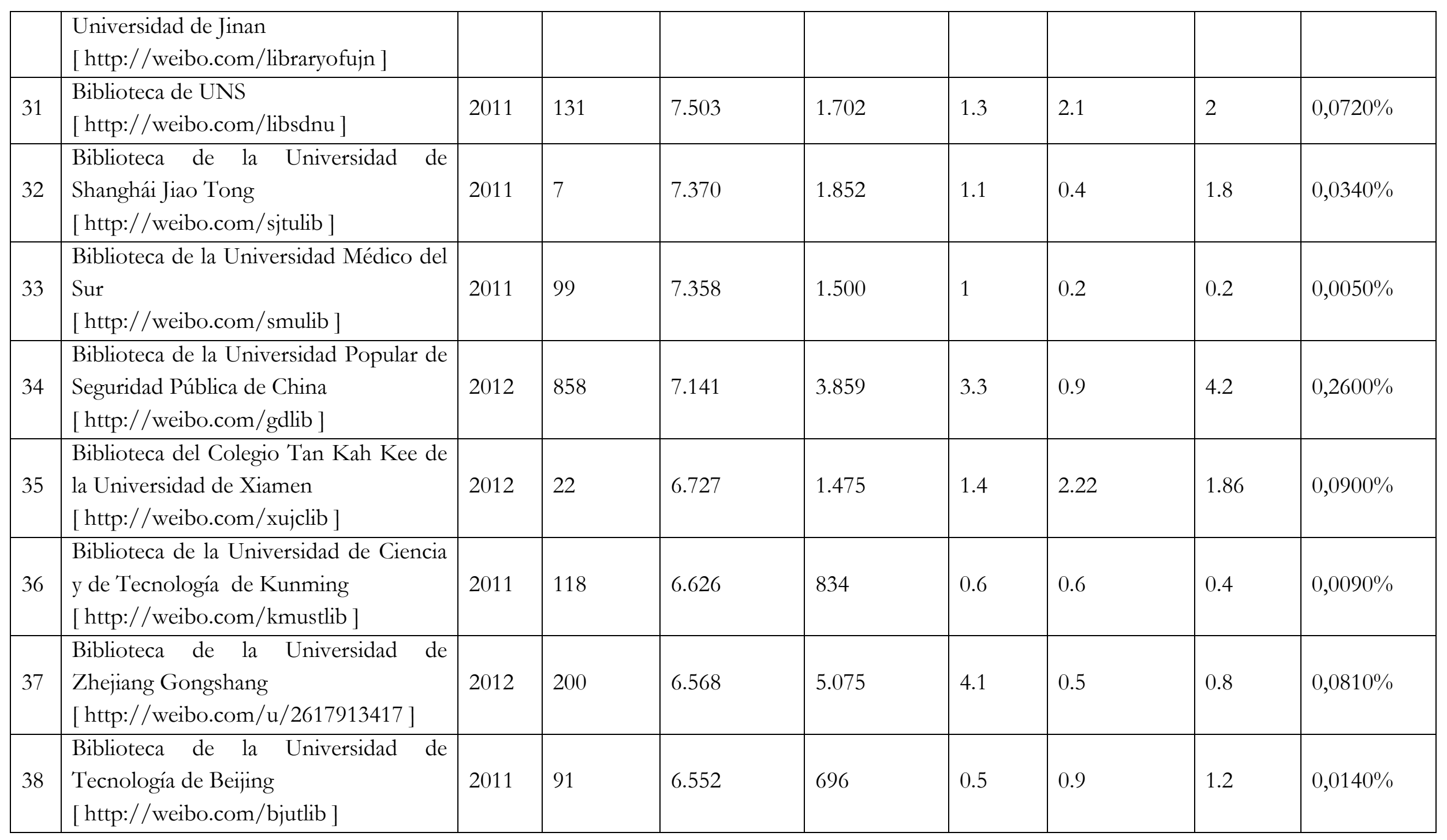




\begin{tabular}{|c|c|c|c|c|c|c|c|c|c|}
\hline 39 & $\begin{array}{l}\text { Biblioteca de la Universidad de Xi'an } \\
\text { Jiaotong } \\
\text { [ http://weibo.com/xjtulib ] }\end{array}$ & 2011 & 17 & 6.479 & 251 & 0.2 & 0.2 & 0.2 & $0,0010 \%$ \\
\hline 40 & $\begin{array}{l}\text { WO de la Biblioteca de la Universidad } \\
\text { de Qingdao Binhai } \\
\text { [ http://weibo.com/u/5135552818 ] }\end{array}$ & 2014 & 1090 & 6.285 & 1.117 & 2.6 & 0.31 & 0.6 & $0,0004 \%$ \\
\hline 41 & $\begin{array}{l}\text { Biblioteca de la Universidad de } \\
\text { Zhengzhou } \\
\text { [ http://weibo.com/zzulib ] }\end{array}$ & 2013 & 74 & 6.149 & 371 & 0.6 & 2.63 & 0.95 & $0,0400 \%$ \\
\hline 42 & $\begin{array}{l}\text { Biblioteca de la Universidad de Hainan } \\
\text { [ http://weibo.com/libhainu ] }\end{array}$ & 2012 & 104 & 6.142 & 2.174 & 2.1 & 2.61 & 1.46 & $0,1400 \%$ \\
\hline 43 & $\begin{array}{l}\text { Biblioteca de la Universidad de } \\
\text { Tecnología y Negocios de Beijing } \\
\text { [ http://weibo.com/tsgbtbu ] }\end{array}$ & 2011 & 202 & 6.042 & 753 & 0.5 & 0.3 & 0.9 & $0,0110 \%$ \\
\hline 46 & $\begin{array}{l}\text { Biblioteca de la Universidad de } \\
\text { Finanzas y Economía de Guangdong } \\
\text { [ http://weibo.com/gdcclib ] }\end{array}$ & 2011 & 285 & 5.638 & 1.986 & 1.3 & 1 & 0.9 & $0,0430 \%$ \\
\hline 47 & $\begin{array}{l}\text { Biblioteca de la Universidad Normal de } \\
\text { Hebei }\end{array}$ & 2011 & 154 & 5.594 & 1.370 & 0.9 & 0.4 & 0.6 & $0,0160 \%$ \\
\hline
\end{tabular}




\begin{tabular}{|l|l|l|l|l|l|l|l|l|}
\hline & $\begin{array}{l}{[\text { http://weibo.com/hbsfdxtsg }]} \\
48\end{array}$ & $\begin{array}{l}\text { Biblioteca de la Universidad de } \\
\text { Tecnología de Hefei } \\
{[\text { http://weibo.com/hfutlib] }}\end{array}$ & 2011 & 66 & 5.526 & 2.584 & 1.8 & 0.1 \\
\hline 49 & $\begin{array}{l}\text { Biblioteca de la Universidad de Ciencia } \\
\text { Política y Derecho del Suroeste } \\
{[\text { http://weibo.com/u/3519535423] }}\end{array}$ & 2013 & 115 & 5.113 & 1.004 & 1.3 & 1.23 & $0,0160 \%$ \\
\hline 50 & $\begin{array}{l}\text { Biblioteca de la Universidad de } \\
\text { Económicas y Derecho de Zhongnan } \\
{[\text { http://weibo.com/u/3313319852 }]}\end{array}$ & 2013 & 73 & 5.101 & 673 & 0.8 & 2.31 & $0,1000 \%$ \\
\hline
\end{tabular}

Anexo 1. Tabla de datos correspondiente a los perfiles de las primeras 50 bibliotecas universitarias chinas en Weibo 